\title{
Preface
}

\section{Intersection Between the Reading Room and the Operating Room}

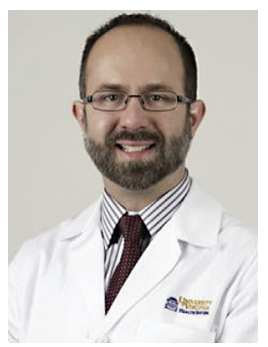

Nicholas C. Nacey, MD

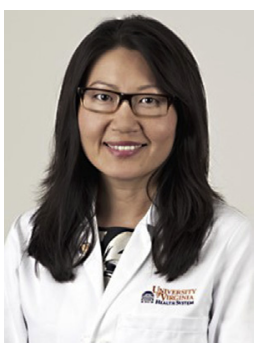

Jennifer L. Pierce, MD

Editors

Orthopedic surgeons and musculoskeletal radiologists both interpret imaging studies as part of routine day-to-day practice. However, these 2 specialties approach image interpretation differently based on the perspective of their particular profession. Mutual understanding of each other's specialties is critical to providing the best patient care that we can. From the radiologist's perspective, we are always intrigued by seeing surgical correlations for the MRI findings that we see on a daily basis and can use knowledge that is gained about the surgical approaches undertaken by our colleagues to inform our understanding of expected postsurgical appearances. For orthopedists, knowledge about different imaging techniques as well as the normal and pathologic appearances of musculoskeletal structures can help to inform what you may be seeing in a patient in front of you in clinic or intraoperatively. We have always been exceedingly grateful for the collaboration we've had with our orthopedic colleagues and the insights we have gained, and as such, we hope to reciprocate by giving the readers a look inside the reading room with a group of leaders in the field of musculoskeletal radiology.

We have decided to take a shoulders-to-toes approach to sports medicine imaging, mixing bread-and-butter topics with others that tend to receive less attention in the literature but are still critical to managing patients with sports injuries. The first 3 articles deal with the upper extremity with focuses on shoulder instability, elbow injuries, and hand/wrist trauma. This is followed by a quartet of articles focusing on the knee with detailed imaging descriptions of the menisci, ligaments, cartilage, and patellofemoral instability. Understanding pathologic condition in the native knee is often only half the battle in image interpretation, and as such, these articles also describe some of the differences when evaluating imaging of postoperative patients. Articles on intraarticular and extraarticular causes of hip pain, tendon and ligament injuries in the ankle, and turf toe round out our tour of the lower extremity. Last are three articles 
encompassing sports medicine imaging as a whole. The article on stress imaging describes findings on different modalities and classifies what particular findings are considered high risk. The next article on pediatric sports injuries highlights the unique pathologic condition found in the pediatric patient population. The final article on musculoskeletal ultrasound describes the growing importance of this modality as well as situations in which ultrasound should be considered for imaging evaluation.

We would like to thank Dr Miller for the opportunity to serve as guest editors, as well as Lauren Boyle, Donald Mumford, and Diana Ang at Elsevier for helping us to put this issue together. Last, we are extremely grateful to the many authors of this issue for contributing their extraordinary expertise; we could not have put this issue together without the assistance of these exceptional colleagues. We hope that the readers of the issue will find that the trip to the reading room was well spent.

Nicholas C. Nacey, MD

Department of Radiology and Medical Imaging

University of Virginia

1215 Lee Street

PO Box 800170

Charlottesville, VA 22903, USA

Jennifer L. Pierce, MD

Department of Radiology and Medical Imaging

University of Virginia

1215 Lee Street

PO Box 800170

Charlottesville, VA 22903, USA

E-mail addresses:

ncn5t@hscmail.mcc.virginia.edu (N.C. Nacey) jp5aq@hscmail.mcc.virginia.edu (J.L. Pierce) 